\title{
La construction des appellations viticoles et la géographie juridique
}

De l'élaboration de limites dans le cadre du projet de hiérarchisation d'une aire viticole, le cas de l'AOC Cahors (Lot), ou comment introduire du droit dans une démarche géographique

The construction of the guaranteed vintage and the legal geography; of the elaboration of limits within the framework of the project of hierarchical organization of a wine-making area, the case of the guaranteed vintage of Cahors (Lot), or how to introduce some law into a geographical approach

\section{Éric Rouvellac}

\section{(2) OpenEdition}

\section{Journals}

Édition électronique

URL : http://journals.openedition.org/developpementdurable/10811

DOI : 10.4000/developpementdurable.10811

ISSN : 1772-9971

Éditeur

Association DD\&T

Référence électronique

Éric Rouvellac, "La construction des appellations viticoles et la géographie juridique », Développement durable et territoires [En ligne], Vol.6, $n^{\circ} 1$ | Mars 2015, mis en ligne le 31 mars 2015, consulté le 21 avril 2019. URL : http://journals.openedition.org/developpementdurable/10811 ; DOI : 10.4000/ developpementdurable.10811

Ce document a été généré automatiquement le 21 avril 2019

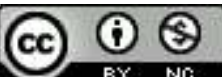

Développement Durable et Territoires est mis à disposition selon les termes de la licence Creative Commons Attribution - Pas d'Utilisation Commerciale 4.0 International. 


\section{La construction des appellations viticoles et la géographie juridique}

De l'élaboration de limites dans le cadre du projet de hiérarchisation d'une aire viticole, le cas de l'AOC Cahors (Lot), ou comment introduire du droit dans une démarche géographique

The construction of the guaranteed vintage and the legal geography; of the elaboration of limits within the framework of the project of hierarchical organization of a wine-making area, the case of the guaranteed vintage of Cahors (Lot), or how to introduce some law into a geographical approach

\section{Éric Rouvellac}

\section{Le contexte des appellations du Sud-ouest de la France}

1 Les appellations viticoles du Sud-ouest de la France, figure 1, souffrent quasiment depuis leur création d'un déficit de réputation, qui a contribué à une certaine mévente historique. Il suffit de regarder la façon dont on classe les appellations françaises habituellement dans les ouvrages de vulgarisation à propos de la vigne et du vin. Elles sont nommées par leur nom (Alsace, Bordeaux, Bourgogne) ou par des noms régionaux évocateurs (vallée de la Loire, vallée du Rhône). Les appellations du Sud-ouest apparaissent non seulement en dernier, mais encore seulement désignées par des points cardinaux, constituant l'ancien bassin d'approvisionnement du port de Bordeaux. Cet éclatement géographique se superpose à une tradition de production de vins améliorateurs tanniques, avant la crise phylloxérique, qui a eu du mal à évoluer, sans doute aussi à cause de l'exode rural au $\mathrm{XX}^{\mathrm{e}}$ siècle qui a abouti à une perte importante des savoir-faire. Leur marché n'apparait alors essentiellement que comme local. La mise en concurrence à tous les nivaux, nationale, européenne et avec les nouveaux pays producteurs a amené les décideurs de la filière vitivinicole, (élus de chambres d'agricultures, dirigeants de syndicats viticoles, ingénieurs ou techniciens), a réfléchir sur 
la manière de faire émerger un socle, utilisable pour le viticulteur et lisible pour le consommateur, faisant apparaitre les différentes qualités de vin d'une appellation. Cela a commencé par des études de cartographie des terroirs initiées par la Chambre régionale d'agriculture de Midi-Pyrénées dans les années 1990 qui ont concerné les appellations viticoles de Cahors, Gaillac, Fronton, Saint-Mont et les vignobles aveyronnais. Plus tard, entre 2001 et 2004, ce fut le tour de la Chambre d'agriculture de Dordogne de diligenter une étude cartographique des terroirs bergeracois. Nous eûmes alors l'occasion de nous occuper des AOC Cahors et Bergerac, (Rouvellac, 2005, 2006). Parallèlement, le Syndicat de défense de l'appellation Cahors s'est préoccupé de mener une hiérarchisation au sein de l'aire AOC, voulant faire émerger de la sorte le meilleur potentiel et les meilleurs vins de l'appellation. Un vocabulaire associé à cette hiérarchisation permettrait également de mieux communiquer et faire connaitre les produits. Il nous fut demandé de réunir les éléments nécessaires à cette hiérarchisation, à savoir mettre en place des critères de différenciations des terroirs en fonction de leurs aptitudes viticoles dans le cadre d'une production à forte valeur ajoutée, et puis cartographier l'ensemble à l'échelle cadastrale, parcellaire.

2 C'est finalement au cours de ce travail, étalé entre 1997 et 2001, que s'est posé avec acuité le problème de l'intervention du droit dans nos recherches à travers la notion de limite. Les implications de notre cartographie étaient potentiellement importantes, tant sur le prix des terres que sur le prix final de la bouteille. Du coup, le tracé de cette hiérarchisation, avalisé par la loi, prenait une autre dimension, où la réflexion géographique créait du droit, où les deux disciplines se retrouvaient côte à côte pour expliquer un territoire.

3 Nous nous proposons de réfléchir à cette imbrication entre géographie et droit en présentant comment les deux disciplines peuvent se combiner, théoriquement et avec l'aide d'un exemple à petite échelle. Puis nous réfléchirons à comment utiliser la notion de limite, comment l'appliquer, comment construire du droit avec l'exemple de la hiérarchisation au sein de l'aire AOC Cahors.

\section{Comment la géographie peut mener au droit}

Depuis la loi de 1905 dite sur la répression des fraudes, puis avec le cadre fourni dans les années 1930 avec la création de l'INAO, caractériser, délimiter, spécifier, différencier, singulariser..., telles sont les missions des experts, des viticulteurs, des techniciens pour construire des appellations ou des hiérarchisations dans le milieu des productions agricoles, à la recherche potentielle d'une forte valeur ajoutée basée sur la qualité, garantissant celle-ci au consommateur tout en valorisant le revenu du producteur. 
Figure 1. Les appellations qui composent les AOC et VDQS du Sud-ouest

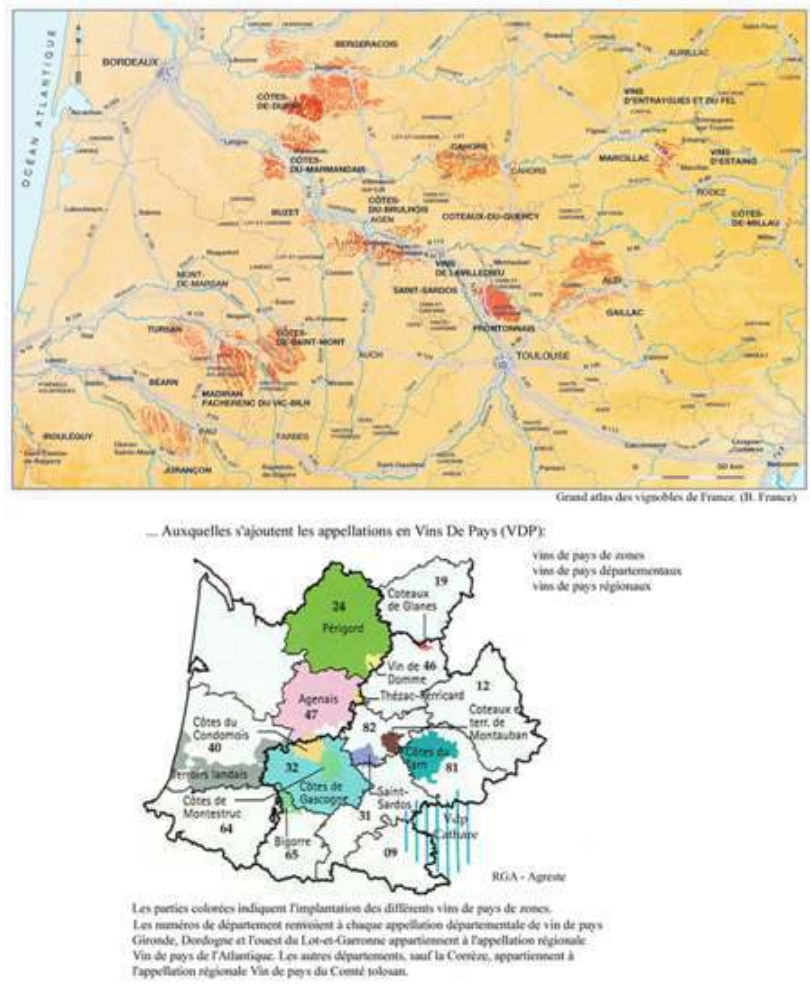

\subsection{La construction des appellations passe par définir des limites}

La volonté de définir des limites à partir de formes de relief héritées est une chose, mais les conséquences sociales et économiques en sont une autre qui ne peut être négligée Ces délimitations donnent une dimension d'aménagement du territoire sur du moyen et du long terme et risquent d'influencer avec force du prix des terres jusqu'au prix du vin. C'est à ce moment là que la juridisation devient nécessaire, que le droit s'introduit dans la géographie. Nous en avons pris conscience durant la cartographie des terroirs des aires AOC Cahors et Bergerac, (Rouvellac, 2005, 2006), dans l'esprit d'un exercice scientifique. Le projet de hiérarchisation de l'aire AOC Cahors, entre 1997 et 2000, (Rouvellac, 2002), nous a fait pleinement prendre acte des conséquences d'un classement des territoires. Élaborer des limites et donc décréter une hiérarchie des parcelles en fonction de qualités potentielles de la production viticole revient à introduire du droit, ou du moins de la norme dans une démarche géographique.

De manière pratique, la cartographie à grande échelle des terroirs des aires AOC Cahors et Bergerac et la hiérarchisation de l'aire AOC Cahors nous ont assez vite amené à des interrogations quant au choix du tracé de la limite entre deux unités. Ces échelles du $1 / 25000, d u$ 1/10000 voire du 1/5000 impliquent parfois une argumentation solide devant les acteurs de la filière vitivinicole, et devant la communauté scientifique aussi. Se posent des questions parfois difficiles, $d u$ choix du passage du trait, brisure et changement brusque, quand dans la réalité parfois seul le continuum et la progressivité font loi... 
7 De plus, le simple choix se double d'une validation juridique; par exemple les limites proposées dans le cadre d'une hiérarchisation en crus dans l'aire AOC Cahors sont susceptibles d'être reconnues par règlementation, en droit. Ceci possède bien sur des implications importantes quant à la reconnaissance et au prix du produit final, le vin issu de ces terroirs, est théoriquement de plus en plus cher au fur et à mesure qu'on monte en gamme, que les vignes se trouvent sur des terroirs définis comme potentiellement de plus en plus qualitatifs.

8 Une réflexion sur la notion de limite et sa mise en forme juridique s'impose alors. Il existe une convergence de perspectives entre la géographie et le droit qui permet de dire le droit à partir d'approches spatiales avec les mots de la géographie et, réciproquement, d'appréhender le spatial avec les mots et la théorie du droit.

9 C'est surtout au cours du travail de hiérarchisation de l'aire AOC Cahors que s'est posé avec acuité le problème de l'intervention du droit dans nos recherches à travers la notion de limite. Les implications de notre cartographie étaient potentiellement importantes, le tracé de cette hiérarchisation, avalisé par la loi, prenait une autre dimension, où la réflexion géographique créait du droit, où les deux disciplines se retrouvaient côte à côte pour expliquer un territoire. Une position où à présent nous occupions autant la place de chercheur que d'acteur du monde vitivinicole.

10 La cartographie de ces terroirs pose le problème de la limite des ensembles retenus en fonction des critères. La nature et son aménagement incessant (jusqu'à l'abandon) par les sociétés aboutissent rarement à des contours d'espaces homogènes précis. Les acteurs doivent tracer des limites nettes là où tout n'est que transition et continuum, d'où l'importance des critères retenus, de l'échelle spatiale sur laquelle repose la réflexion, de la mise en forme juridique qui va asseoir cela.

11 Nous nous sommes proposés de réfléchir à cette imbrication entre géographie et droit à travers l'exemple de la hiérarchisation au sein de l'aire AOC Cahors, tout d'abord en présentant comment les deux disciplines peuvent se combiner, ensuite nous essaierons de développer l'exemple qui nous a été donné d'analyser.

La recherche géographique, ici d'une mise en équation du réel, d'une modélisation, sans tomber dans la philotomie, est mise face à la démarche abstraite du juriste, qui cherche lui aussi à coder la réalité, mais dans un but plus simplificateur pour l'usager, le citoyen, (Perret, 1994, Forest, 2009). La différence réside dans le fait que le géographe s'attache à faire transparaitre, tant dans ses démarches que par la cartographie, les nuances, les transitions, les continuums qui ne cessent de compliquer le réel. Alors que le juriste est chargé de faire appliquer un règlement basé sur la notion de limite, nette et précise, entre deux ensembles; dans l'un s'exerce un droit qui n'existe pas dans l'autre. Le droit fige alors des périmètres pour que la société puisse en faire plus facilement l'usage qu'elle a décidé.

Dans le cas de la création d'une aire apte à fournir un produit agricole reconnu et qualitatif, élaborer des critères pour définir une aire, une appellation cohérente, physiquement, économiquement, culturellement nécessite la création d'outils scientifiques et juridiques, visant à mettre un ordre et sortir de la confusion, à tendre vers une objectivité dans le réel, l'environnement, l'histoire, les aspects socioéconomiques pour donner des arguments au juridique qui va légiférer sur le sujet. En créant des limites, il se crée de l'ordre mais il se perd en nuance, on participe à construire aussi le réel. 

géographique, amenant des interactions entre géographie et droit, une juridisation de la géographie ou une "géographisation » du droit. Il faut donc construire des normes, du droit, pour caractériser un espace apte à être reconnu, en fixer les limites en déterminant des critères, ce qui va revenir à donner une identité à ces portions d'espace, à ces terroirs. Les méthodes utilisées pour la construction de normes juridiques à partir de modèles géographiques peuvent s'appuyer alors sur ces batteries de critères tant physiques, économiques, sociaux que culturels. Le droit peut alors aboutir à un construit social pour un contrat du même nom, (Melé, 2009).

Cette notion de limite se veut objective, on cherche à l'objectiver pour qu'elle ne soit pas remise en cause, et pour cela le juriste et puis le législateur s'appuient sur des phénomènes naturels, (qui parfois sont déjà hérités, et du coup fort peu naturels). Si les limites ne sont pas bien fixées, par l'usage et le droit, elles peuvent être mises à mal par des intérêts particuliers, peu soucieux de démarches collectives permettant de fabriquer un tissu économique plus distributif. Les experts s'appuient alors sur les notions d'usages locaux, loyaux et constants et sur la pérennité qui peu à peu participent à fixer de la norme, de l'habitus (c'est-à-dire ici une manière de cultiver la vigne et d'élaborer du vin), ce qui ensuite construit du droit, des valeurs jurisprudentielles.

Ceci aboutit à transcrire de la norme (technique et sociale) dans le droit, avec l'écueil sans cesse présent d'aboutir à des espaces trop statiques. La construction d'un espace apte à produire une appellation contrôlée fige le territoire d'une certaine façon, créant des relations en sens unique car tournées vers une seule production. Cela induit de nouvelles relations, de nouvelles expériences territoriales, un cadre de nouvelles sociabilités.

Il devient alors intéressant d'étudier comment la géographie interagit sur le territoire, sur la définition des terroirs, par l'interprétation du réel, (naturel ou pseudo naturel, économique et social), par un rôle d'expertise, d'examen des jeux d'acteurs.

\section{2. À l'échelle de communes, comment construire une appellation et la valider juridiquement ?}

Dans le cas qui nous préoccupe, pour définir des aires viticoles potentiellement qualitatives pour le vin, ou bien construire des nuances à l'intérieur de celles-ci pour hiérarchiser ces potentiels, il nous faut un point de départ, une définition de «l'aire de jeu » en quelque sorte. D'où les consultations des conseils municipaux par les préfectures pour savoir quelles communes veulent rentrer dans cette construction territoriale. C'est donc en s'appuyant sur la plus petite entité administrative, aux limites bien établies par les usages et la loi, que les experts vont procéder. Et de fil en aiguille, les limites cantonales, départementales, régionales vont concourir à définir un premier périmètre avec l'accord des producteurs, des élus, des experts.

Il y a des communes qui arrivent, par entregents et influences, à se faire rattacher à une appellation voisine à l'origine plus prestigieuse que celle où elle devrait figurer. C'est le cas de Salsigne dans l'Aude, située dans l'appellation Cabardès, et qui a réussi à faire partie du Minervois voisin à l'est, en arguant que les terrains correspondaient plus à ce dernier. Cet argument naturaliste encore une fois à postériori cache (mal) une situation sociale particulière de la commune de Salsigne d'alors. Au début des années 1980, (l'AOC Minervois date de 1985), La commune abrite encore une mine d'or en activité, les mineurs 
sont des gens possédant un fort poids social localement, qui ont du pouvoir d'achat et qui sont à leurs moments perdus viticulteurs en complément d'activité. Ils estiment alors que leur commune ne doit pas être écartée de cette AOC voisine, mais ne la jouxtant pas, et obtiennent gain de cause. Salsigne est maintenant une enclave du Minervois dans le Cabardès, devenu AOC en 1999, figure 2.

Figure 2. $\mathrm{L}$ cas de la commune de Salsigne, faisant partie de l'appellation Minervois dans l'Aude, esseulée à gauche, constituant un hiatus à droite dans l'appellation Cabardès

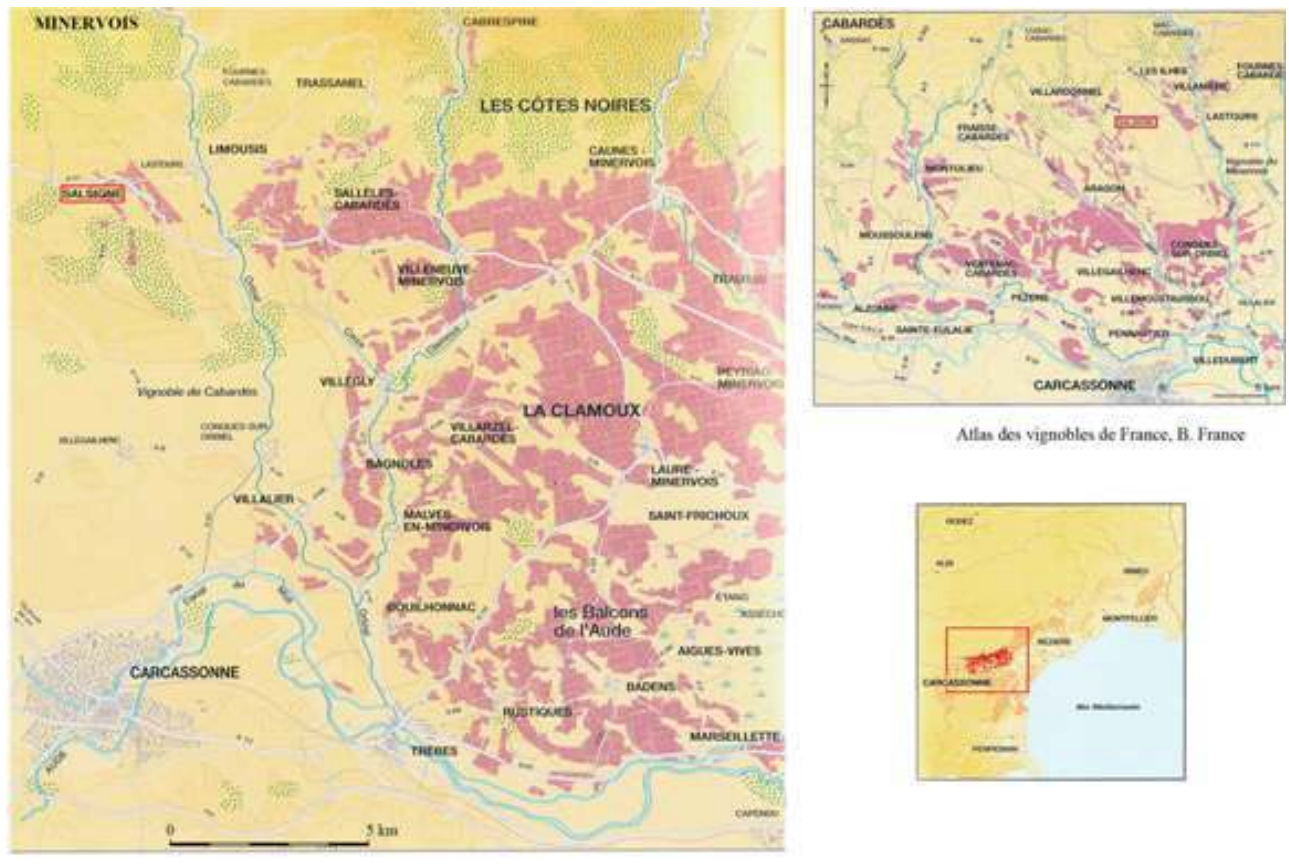

S'avance dans ce premier moment de l'élaboration d'une AOC (ou de tout autre produit labellisé), le problème d'asseoir une production agronomique, dépendante en partie non négligeable de facteurs naturels et aussi sociaux, au sein d'un ou de territoires administratifs, de gestion, dont les limites s'affranchissent de la nature, reposant sur des accords politiques et culturels de longue haleine. Nous pouvons prendre à ce sujet les exemples des aires AOC Cahors et Bergerac encore une fois, figure 3. À l'échelle communale, il apparait des hiatus dans la distribution des communes qui composent le support de l'aire contrôlée. Comment expliquer qu'à coté de la commune de Caillac, une encoche montre l'omission des deux communes d'Espère et de Calamane? Mêmes terroirs, même histoire, et pourtant le choix des municipalités, qui remonte à la construction du VDQS en 1951, a été de ne pas rentrer dans la construction d'une production viticole à plus forte valeur ajoutée et plus forte lisibilité. C'est la même chose quand on examine le trou que forme deux communes au cœur de l'AOC Bergerac juste à l'ouest de la sous préfecture. Ici, les limites communales ont forgé les territoires viticoles, et même au-delà. Au sein des communes comportant des parcelles AOC, le prix du terrain viticole peut atteindre jusqu'à 15 fois supérieur à la moyenne départementale ${ }^{1}$. Les terrains sont forcément plus recherchés dans la zone AOC, où le potentiel économique est plus fort (si le marché est propice) et la déprise gagne plus facilement les communes limitrophes qui ont refusé de rentrer dans l'aire, ce qui aboutit à des modifications sur la longue durée dans lesquelles les aires AOC participent à structurer les paysages. 
Figure 3. Situation des aires d'appellation d'origine contrôlée de Cahors et de Bergerac

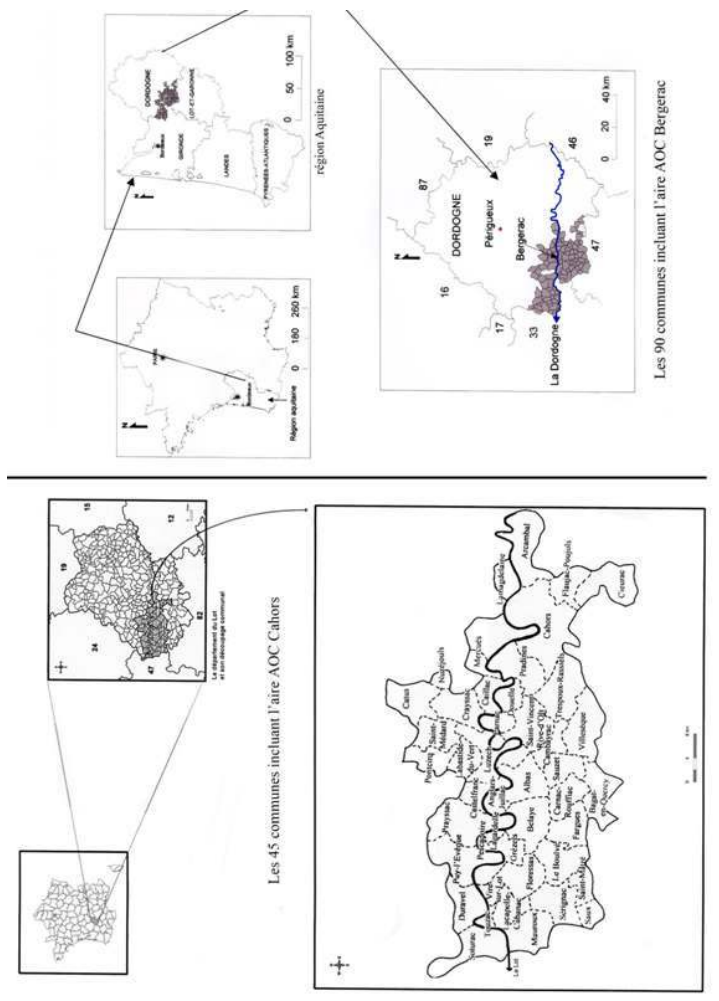

Face à ces municipalités qui ont fait le choix, non rétroactif, de ne pas faire rentrer leur territoire dans la construction d'une appellation, avec une vision plutôt "pessimiste", s'observent des «opportunistes ». Ce sont des communes où l'usage viticole n'était déjà pas bien ardent au moment de la construction de l'aire, et où parfois il s'est éteint par la suite, mais dont le conseil municipal a choisi de rentrer dans des démarches de labellisation, ne voulant pas se couper d'un potentiel de développement par la suite. Ainsi apparaissent les communes du nord de l'appellation Cahors, ou du sud-est des appellations Bergerac.

Dans ces trois cas, (Minervois, Cahors, Bergerac), les limites liées aux facteurs naturels sont sans objet, seule la question de limites sociales et culturelles peut être évoquée. La limite de l'aire est alors forgée par l'expression des élus ou d'un groupe de pression qui s'appuie sur des élus. La limite communale sert d'appui au droit pour réglementer des niveaux de production viticoles qui auront des conséquences économique et sociales fortes. Nous sommes loin du terroir.

\section{Comment la géographie sert la réglementation : l'exemple de la hiérarchisation de l'aire AOC Cahors}

Si l'on s'intéresse à des échelles plus grandes pour comprendre encore le rôle de la limite dans la construction du droit appliqué aux appellations, seules des échelles de l'ordre du $1 / 25000$ jusqu'au 1/5000 parfois peuvent être choisies dans la volonté de trouver des critères discriminants, et donc des limites les plus fiables possibles, les plus justifiables. D'où la nécessité de définir des critères culturaux et culturels au plus près du terrain, d'aller loin dans le détail. 

niveau recherché. En effet le principal facteur limitant de ce type de terroir reste les sols, essentiellement limono sableux, donc filtrants, ils ne possèdent pas assez de réserve hydrique pour alimenter la vigne en cas de sécheresse. Ces sols demeurent peu lessivés, riches naturellement en nutriments et oligoéléments, ce qui ne favorise pas l'enracinement profond et maintient des rendements trop élevés, trop diluant qualitativement. A contrario, s'il pleut trop, l'absence de pente de ce terroir va aboutir à une stagnation de l'eau en surface néfaste à l'endroit de quelques affleurements plus argileux. Il ne faut pas négliger que localement, au gré des dépôts de la rivière, peuvent affleurer des lentilles de graves plus argileuses, aux sols pouvant à la fois garder l'eau et se ressuyer, constituant des parcelles intéressantes pour une viticulture de qualité. Seul un examen minutieux, à très grande échelle et au cas par cas, peut révéler des terroirs d'exception dans cette moyenne terrasse.

Par exemple, selon l'expérience des viticulteurs, un simple palier de terrasse de 1 à 1,5 m de commandement est reconnu avoir une influence sur le développement de la vigne et la qualité du raisin : en contrebas, il y a risque de gelée tardive, accumulation d'air froid et d'humidité, le terroir peut être plus tardif et moins qualitatif, en contrehaut, apparaît une partie dégagée de ces mauvaises influences et plus qualitative, le tout sur une distance de quelques mètres. Tracer les limites des différents terroirs définis par ce palier de terrasse est possible à l'échelle cadastrale, la réglementation qui peut en découler s'appuie ici sur un phénomène ténu, mais aux conséquences importantes, puisque ce palier ou ce talus de terrasse peut délimiter des niveaux alluviaux de différent potentiel vitivinicole pour les experts.

Mais parfois ce trait ne trouve aucun repère visible ou s'accrocher, sa justification en est encore plus malaisée, d'où la recherche de seuils parfois empiriques pour trouver une limite.

défense de l'AOC Cahors, des viticulteurs, de l'INAO pour hiérarchiser l'appellation afin d'introduire une démarche qualitative, de déterminer le meilleur potentiel viticole à l'intérieur de l'aire AOC. Nous avons opéré dans un contexte de mévente, de réputation usurpée, de piètre qualité généralisée, mais avec une volonté de tirer l'appellation vers plus de qualité en valorisant les meilleurs potentiels. Il n'est pas du tout question de retirer le droit à certains de produire du raisin sous prétexte que leurs parcelles seraient seulement « mal situées ».

de l'appellation à travers les terroirs mettrait en évidence le meilleur du potentiel vitivinicole, mettant en adéquation ce zonage avec le marché, le prix du vin avec une qualité construite par le viticulteur, qui peut s'appuyer sur le potentiel des terroirs. Prenons le cas de la deuxième terrasse par exemple :

Selon nos critères, il n'y a pas de hiérarchisation possible et peu de potentiel qualitatif du politique des forts rendements au détriment de la qualité générale des vendanges. On a alors remplacé petit à petit la fruiticulture, les céréales, le tabac, l'élevage par le vignoble. La périurbanisation ou le mitage n'étaient pas des phénomènes très prégnants et ont touché en premier les terroirs de la haute terrasse, bien exposés et aux sols plus pauvres, plus lessivés. 

constituant presque $25 \%$ du vignoble; serait-ce bien raisonnable? Les aspects socio économiques vont alors primer sur le raisonnement scientifique. De toute façon, il est possible de produire des vins d'une certaine qualité sur ce type de terroir; il existe un marché en dehors des crus, par définition rares et onéreux.

Ce sont les viticulteurs avec l'impulsion de leur Syndicat de défense qui ont décidé, à partir de 1997, de construire une hiérarchisation de l'aire AOC, sous la forme d'une pyramide allant vers plus de qualité en grimpant vers le sommet. Cette démarche a été avalisée par l'INAO, l'autorité ministérielle de tutelle des AOC. Le syndicat s'est alors tourné vers nos services pour que nous élaborions en commun avec les viticulteurs les critères de cette hiérarchisation, en partant de la carte des terroirs que nous avions construite. À la fin de notre travail, le Syndicat devait le valider ou non : dans le premier cas, il appartenait alors à l'INAO de se prononcer à son tour pour lancer ou non le processus de hiérarchisation au niveau juridique.

Nous avons commencé une première série de réunions avec les viticulteurs où nous avons élaboré des critères généraux de hiérarchisation dans la vallée du Lot, sur les plateaux bordiers. Les viticulteurs ont été interrogés pour savoir où ils situaient empiriquement les meilleurs ou bien les plus mauvais terroirs. Nous avons décidé aussi ensemble de la forme juridique que cela prendrait (une pyramide de hiérarchisation) et nous avons défini les superficies qu'il convenait de ne pas dépasser pour rester viable économiquement. Nous devions faire en sorte qu'il n'y ait pas une inflation de demandes de classement en crus afin qu'elles ne prennent pas le pas sur le potentiel économique et le raisonnement scientifique. Les viticulteurs se sont d'ailleurs mis d'accord pour que seulement $10 \%$ de l'aire AOC, 2170 ha sur $21700 \mathrm{ha}^{2}$, soient potentiellement productive en "sous régionale $"$, figure 4.

Après plusieurs mois d'examen des parcelles AOC du vignoble, nous sommes retournés devant la profession lors d'une deuxième série de réunions pour montrer ce que donnait le premier étage du classement, la « sous régionale ». 


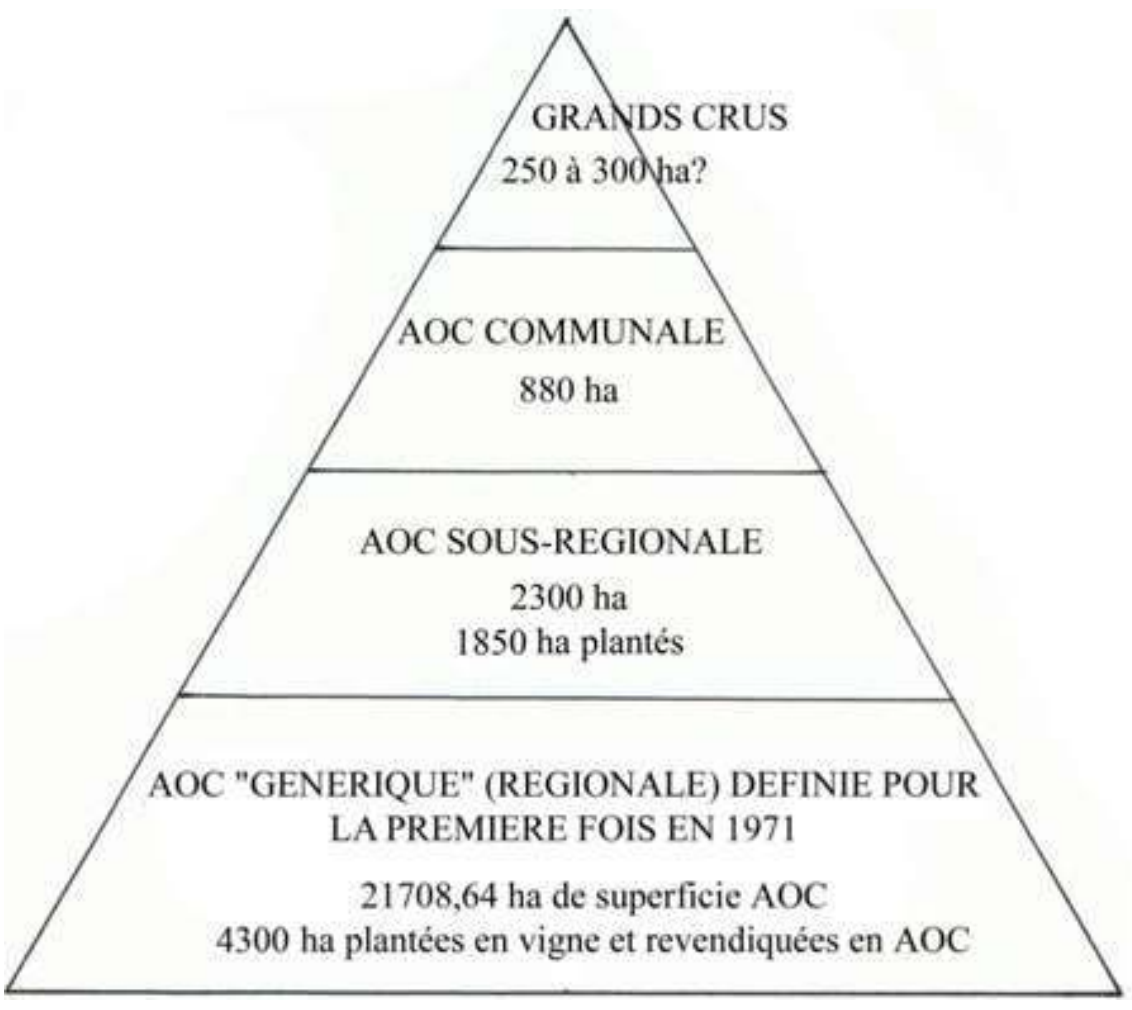

La délimitation parcellaire globale proposée en "sous régionale » représentait environ 1850 ha, dont 1500 ha en vigne et 350 ha en jeunes vignes ou terrains nus, soit $8,5 \%$ de l'aire AOC Cahors actuelle, mais $44 \%$ de l'aire AOC réellement viticole. Cette proposition de délimitation a été approuvée pour $99,7 \%$ des parcelles proposées par l'ensemble des vignerons à l'exception de 7,65 ha, (propositions de classement refusées). Les demandes de classement complémentaires par les viticulteurs concernent 430 ha, soit $2 \%$ de l'aire AOC Cahors, mais $24 \%$ de l'aire " sous régionale » proposée.

Ces demandes complémentaires concernent deux types de terroirs, la moyenne terrasse et les plateaux de calcaires lacustres, tous deux exclus de nos critères de choix, en accord au départ avec les viticulteurs. C'est à l'INAO et ses experts de trancher ce problème.

Nous sommes passés ensuite à un classement en "communales ", deuxième étage de la hiérarchisation, pour les six communes du noyau d'élite de l'appellation, approuvé lui aussi par les viticulteurs. Ce sont 884 ha qui sont susceptibles de figurer à ce niveau, dont une grande proportion est déjà plantée, le durcissement des critères ne permettant pratiquement pas de proposer des terrains vierges de vigne.

37 Nos travaux se sont arrêtés là, le Syndicat de défense et l'INAO ont accueilli très favorablement notre méthode et nos résultats, mais nous n'avons pas poursuivi au niveau des crus. Le Syndicat de défense de l'aire AOC doit d'abord s'accorder avec l'INAO sur les noms que pourrons porter ces différents niveaux, les experts nationaux demeurant réservés sur la possibilité d'employer le mot "cru», réservé implicitement aux appellations majeures du pays.

Au sein du niveau de la "sous régionale ", pour mettre en évidence le meilleur potentiel de l'aire AOC valorisable en crus, il nous a fallu partir de critères environnementaux, sociaux, historiques, économiques, issus de l'étude des terroirs qui la composent. 
39 Nous avons choisi d'éliminer les terroirs alluviaux de la plaine inondable, de la basse terrasse et de la moyenne terrasse, ces terroirs présentant des sols jeunes, donc des sols riches, fertiles, où la nappe phréatique est souvent peu profonde, créant des risques d'hydromorphie, (la vigne ayant besoin d'un régime hydrique naturel et régulier, qui a au moins autant d'importance que la structure du sol dans lequel elle pousse). La texture de ces terrains est souvent à majorité limono sableuse, ce qui implique un sol trop filtrant ou trop battant et donc un déficit ou un excès d'humidité. En cas d'excès, sur les sols mal drainés naturellement, l'eau a tendance à stagner. Or, si la vigne résiste très bien à la sécheresse, en contrepartie, elle craint particulièrement un séjour prolongé dans l'eau.

À partir de la haute terrasse plus érodée, apparaissent des nuances de coteaux et des sols beaucoup plus argileux. Dès qu'un terrain est en pente, l'excès d'eau s'élimine naturellement, les racines de la vigne descendent très loin. Dans des conditions, la vigne supporte les aléas climatiques et leurs effets néfastes en sont gommés. C'est dans les années défavorables que la supériorité de ces coteaux est la plus parlante, en raison de la qualité de leurs sols bien structurés, qui présentent toujours une forte perméabilité et une bonne aération. En conséquence, nous avons retenu comme terroirs aptes à une hiérarchisation le talus entre moyenne et haute terrasse, la haute terrasse, les grèzes et cônes d'éboulis calcaires, les hauts niveaux alluviaux du Quaternaire ancien. Cette démarche restrictive est confortée par l'ensemble des opinions viticoles en réunions communales, où globalement l'aire de futurs crus ne peut aller que dans un sens restrictif par rapport à l'aire AOC Cahors. Au sujet de la hiérarchisation dans la vallée alluviale, voir la figure 5 qui présente un exemple avec la commune d'Anglars-Juillac.

41 Sur les plateaux, le critère le plus discriminant avec l'exposition et la configuration de l'ombre reste l'épaisseur du sol. Seul le plateau sidérolithique possède un sol qui demeure assez profond. Le reste des plateaux calcaires demeure pauvre en épaisseur de sol et donc en réserve hydrique suffisante pour la vigne. Ce caractère étique des rendzines de plateau, accentué par l'érosion due à la pression agricole passée, conduit parfois à un véritable lithosol. Cette logique de sol trop mince est aggravée par la mosaïque végétale de friches et de bois qui entoure et enserre le vignoble des plateaux, lui faisant perdre le plus souvent toute unité. Bien des parcelles, parfois intéressantes pour notre démarche de hiérarchisation, se retrouvent irrémédiablement isolées, parfois en plus à l'ombre des bois. Ce qui amène de l'humidité néfaste à la vigne, ainsi que du pourridié, dans les parcelles défrichées et dessouchées, qui apparaît et tue les ceps dans l'auréole d'arrachement des chênes. Parfois, le sol est artificiel, obtenu par broyage de la rochemère calcaire. Un sol très mince, inférieur à 15 ou $20 \mathrm{~cm}$ d'épaisseur, est alors obtenu du lithosol original, saturé en cailloux, où la fraction fine demeure quasi absente, où le développement de la chlorose par libération du calcaire actif consécutivement au broyage devient un facteur très limitant.

Sur les plateaux ont été exclus les terroirs comportant des marnes grises du Miocène, compte tenu de la chlorose qu'elles occasionnent sur la vigne et au cépage Cot noir qui y demeure très sensible, impliquant des traitements de sulfate de fer, et d'engrais foliaires à base de fer. 


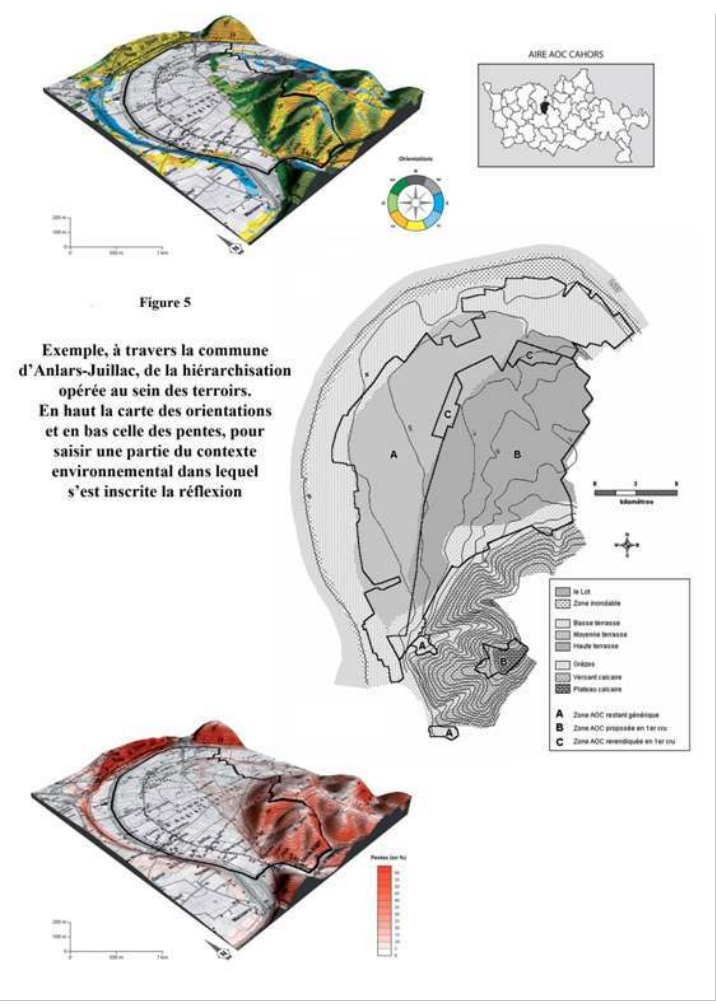

43 Tous les autres terroirs sont susceptibles de propositions de classement à condition de répondre à la même grille de critères physiques :

- les expositions nord et nord-est ont été exclues, constituant un facteur limitant pour le développement de la plante et de ses fruits, par manque de rayonnement solaire ; ce sont en général les terroirs les plus tardifs ;

- les parcelles entourées par des bois sur plusieurs côtés, sans bande sanitaire pour prévoir l'ombre envahissante, ne sont pas proposées ;

- toutes les topographies en creux favorisant les gelées de printemps par accumulation d'air froid sont exclues, comme les fonds de vallées drainées ou sèches et les dépressions fermées.

Les critères liés à l'exposition, sur les plateaux comme dans la vallée du Lot, demeurent les mêmes ; seules seront proposées les parties les mieux dégagées. Tout ce qui peut se trouver à l'ombre trop longtemps dans l'année végétative (à l'ombre des bois environnant essentiellement) est exclu. Une fois ces parcelles-là écartées, seules les expositions sudest, sud, Sud-ouest, ouest et nord-ouest seront susceptibles d'être examinées.

Notre souci a toujours été de proposer un classement par unités assez étendues, seuls des groupes de parcelles ou des parcelles isolées suffisamment grandes ont été proposés. Il est en effet difficile de définir une aire de premiers crus composée de petites parcelles éparses.

47 Les parcelles boisées, en friches ou en polyculture ont été écartées, c'est ici que la notion d'usage et d'antériorité prend tout son sens. Le Quercy a été vidé de ses habitants, la friche a gagné, et l'emboisement a été de grande envergure. La déprise rurale qui s'exerce depuis près d'un siècle et demi amène un changement complet de paysage. Les bois et les friches constituent près de $50 \%$ des 21708 ha de l'aire AOC. Le vignoble, quand il existe, reste le plus souvent extrêmement morcelé sur les plateaux, enchâssé dans les bois et les 
friches, à l'ombre et sans unité. Ce constat ne peut que nous pousser à être rigoureux quant au choix à opérer. En conséquence : pour extraire des argiles de décalcification et des grèzes et les remonter sur le plateau, afin d'introduire une légère épaisseur artificielle de 20 à $30 \mathrm{~cm}$ aux sols dégradés. De cette façon, certains essayent de rehausser le sol au fond des dolines ou de vallées sèches elles aussi gélives. Ce travail ne gomme pas du tout l'effet creux à gelées de ce type de site de terroir. Il faudrait rajouter une épaisseur de plusieurs mètres de terre qui poseraient des problèmes insurmontables de tassement, de glissements de terrain et d'érosion. De plus, rajouter de la terre mêlée à la roche-mère calcaire prise en profondeur libère du calcaire actif et facilite la chlorose. Enfin, ce type d'apport pose une question déontologique à propos de ce qu'on entend par terroir. A-t-on le droit de le modifier à ce point? Le produit élaboré dans de telles conditions est-il fidèle à une origine "naturelle » et invariable comme on l'assure au consommateur dans le cadre d'une AOC? Les viticulteurs de plusieurs régions viticoles se posent ce genre de questions, notamment en Champagne et en Bourgogne. Dans cette dernière, il est admis de ne pas modifier l'épaisseur du sol de plus de $30 \mathrm{~cm}$, en apport ou en excavation.

52 Au sujet des " communales ", sur les plateaux, la logique plus restrictive qui nous guide alors est celle de proposer à une hiérarchisation uniquement les sommets ou lanières de plateau les mieux dégagés des bois et des combes, les mieux exposés, au sol relativement épais, plus de 30 à $40 \mathrm{~cm}$, sans facteur limitant lié au pouvoir chlorosant et ayant une tradition viticole encore existante. Cette logique liée aux terroirs de plateau veut que pour une exposition mauvaise, (composée du nord et/ou de l'est), on retienne la bonne de l'autre côté. Mais c'est justement sur ces parties sommitales du plateau que l'érosion opère son travail de dégradation des sols; et souvent ces parties les plus remarquables possèdent des conditions de sols rédhibitoires à nos critères par leur minceur. La vigne est donc souvent plantée là où le sol est assez épais, mais cette configuration ne se retrouve qu'en bas de pente, soit dans des combes, soit dans des dolines, toutes gélives. Cette conjonction de faits conduit à des propositions très limitées dans les terroirs de plateau calcaire; la notion d'espace dégagé allié à une épaisseur de sol suffisante sera finalement plus importante que celle d'espace élevé, où les bois peuvent toujours être présents et le sol souvent absent. 
53 Toujours au niveau des «communales », mais dans la vallée du Lot, nous nous sommes attachés à garder seulement les meilleures expositions, soient sud, Sud-Ouest ou à plat, les expositions ouest n'étant proposées que rarement à condition que d'autres conditions optimales soient respectées.

54 Seules les parcelles composant les parties les plus hautes des croupes de graves de la haute terrasse et des hauts niveaux alluviaux du Quaternaire ancien sont proposées dans ce nouveau niveau de hiérarchisation. Il nous a paru sage de ne retenir que les endroits les plus plats, les plus hauts, les plus dégagés des phénomènes d'ombres et de gelées et les mieux drainés.

En conséquence, toutes les topographies en creux ou mal exposées, fonds de vallée, dolines, versants, talus et tombant exposés est, sud-est, nord-est, nord-ouest et ouest sont exclus de nos propositions avec plus de sévérité que pour le niveau inférieur. Par exemple, les parties à l'amont d'une vallée sèche disséquant la haute terrasse ont été sorties du nouvel étage de hiérarchisation tant qu'une topographie plane et bien dégagée des courants d'air froid n'est pas retrouvée. Autre exemple, les talus entre moyenne et haute terrasse exposés ouest ne sont pas proposés à ce niveau, car trop à l'ombre en début de journée.

56 Pour les terroirs de grèzes (cône d'éboulis calcaire), le critère de l'exposition sera discriminant, ceux ci restant doté d'une pente non négligeable, assurant le contact entre les niveaux alluviaux et les versants calcaires du causse. Par conséquent, les grèzes situées en rive gauche du Lot coulant d'Est en ouest se retrouvent exposées au nord et au nord-ouest dans leur ensemble; ce qui ne permet plus de les retenir à ce niveau de hiérarchisation.

57 Cette somme de détails de critères qui aboutit à construire des îlots de production potentiellement à forte valeur ajoutée montre comment la méthode s'est enrichie au fur et à mesure de l'avancée du travail. Ce travail montre aussi comment se construisent les opérateurs de jugement définissant une qualité, une réputation, comment la géographie peut mener à une juridisation du territoire, et aussi donc à lui conférer un sens. Si cette hiérarchisation voit le jour, nous aurons construit des normes juridiques à partir de modèles géographiques.

\section{Conclusion}

Le droit n'avalise que des constructions humaines, avec parfois des faiblesses. C'est peutêtre aussi par des aspects en négatifs que nous pouvons saisir le poids d'un découpage territorial au niveau juridique. À Cahors par exemple puisque c'est celui que nous connaissons le mieux, mais nous l'avons constaté dans chaque AOC que nous avons étudié, le classement en AOC originel de 1971 demeure parfois peu rationnel au regard des critères que nous avons mis en avant. Ce qui peut faire comprendre que le secteur vitivinicole ait désiré que son territoire d'appellation évolue. Sans connaitre les tenants et les aboutissants de la construction des limites parcellaires de l'AOC, le spécialiste ne peut être que surpris par des décisions d'inclure des portions de territoire ne correspondant à aucun critère positif. Il semblerait même parfois que dans quelques endroits isolés, ce soit une anti appellation, rassemblant les plus mauvais terroirs, qui soit proposée. Ce sentiment est poussé par exemple par l'ampleur des terrains issus des calcaires et marnes miocènes chlorosantes inclus dans l'aire AOC. Il faut descendre dans l'aval plateau des 
bassins versants pour trouver les affleurements oligocènes dégagés par l'érosion, plus propices au Cot. Les limites étaient respectées empiriquement sur la carte de l'extension du vignoble au XIXème siècle, seuls environ $20 \%$ de la vigne y reposaient, et elles regroupaient surtout des cépages en vins blancs. La volonté d'investir, avec l'avènement de l'AOC uniquement en vin rouge, a été plus forte que les aléas agronomiques et la possibilité d'écouler le vin produit.

Parfois, et dans certaines communes de façon systématique, sont incluses dans l'AOC des parcelles cumulant tous les critères négatifs: roche mère chlorosante, expositions dominées par le nord et/ou l'est, trop exposées aux gelées tardives, aujourd'hui trop à proximité des bois générateurs d'ombre et d'humidité ou carrément abandonnées à la friche ou au bois. Parfois l'appellation apparait sous l'aspect d'une mosaïque de groupes de parcelles éparses, sans logique. Souvent le classement AOC descend dans l'ensemble de la zone inondable du Lot, dans les jardins à l'intérieur des centres bourgs, dans tous les fonds de vallées secondaires, même drainées, sur tous les versants, sauf sur les falaises du Lot qui flirtent avec la verticalité, sur toutes les collines, sans distinction d'expositions. Nous avons là de bonnes illustrations de l'oubli de l'intérêt général au moment où l'AOC a été délimitée pour la première fois et des conséquences que cela va engendrer dans la durée. Ce découpage à l'encontre du potentiel qualitatif du vin local a été avalisé par le droit, a acquis de la légitimité et sera d'autant plus difficilement remis en cause par la suite.

À travers cet exemple de hiérarchisation dans l'appellation Cahors, il nous paraissait instructif d'analyser des convergences de perspectives entre la géographie et le droit. Montrer comment se construit un dossier à finalités juridiques à l'aide d'arguments socio économiques et environnementaux permet d'examiner les articulations entre la géographie et le droit, puisque ce dernier structure aussi notre environnement. Le droit peut alors s'appréhender grâce à la réflexion géographique et constituer des relations sociales, économiques. Il participe à la gestion des ces espaces potentiellement aptes à produire des vins de qualité.

Dans une tentative d'objectivation, le milieu vitivinicole définit donc en partie la qualité par le droit en bâtissant des systèmes d'appellation, mais la qualité passe aussi par une vision biochimique des terroirs. Ceci dans l'optique de pouvoir reproduire de façon pérenne des vins afin de satisfaire le consommateur contemporain, de dégager suffisamment de marge bénéficiaire chaque année pour faire face aux emprunts, aux investissements. Beaucoup de scientifiques ont été sollicités pour cartographier ces potentialités viticoles, pour rendre le concept opérationnel, (Vaudour, 2003).

\section{BIBLIOGRAPHIE}

Forest P., 2009, Géographie du droit, épistémologie, développement et perspectives, Québec, Presses de l'Université de Laval.

Melé P., 2009, « Pour une géographie du droit en action », Géographie et cultures, nº 72, p. 25-42. 
Perret J-M., 1994, « Pour une géographie juridique », Annales de géographie, n 579, p. 520-526.

Rouvellac E., 2001, « Une analyse des paysages de l'aire AOC Cahors : quels enjeux et quelle gestion face à l'enfrichement et à la périurbanisation ». Colloque action paysagère et acteurs territoriaux, 7-8 décembre 2000, Poitiers, GESTE, $\mathrm{n}^{\circ}$ 1, p. 77-87.

Rouvellac E., 2002, « De la cartographie des terroirs de l'aire AOC Cahors à la proposition d'un classement de terroirs en un "premier cru Cahors" ", Norois, n 189, p. 17-31.

Rouvellac E., 2005, Les terroirs du vin de Cahors, Limoges, PULIM (Presses Universitaires de Limoges).

Rouvellac E., 2006, « Les terroirs de l'aire AOC Bergerac », Colloque $\mathrm{VI}^{e}$ congrès international sur les terroirs viticoles, 2-7 juillet 2006, ENITA de Bordeaux, Syndicat AOC Coteaux du Languedoc, Bordeaux, Montpellier, 2 volume, p. 491-496.

Vaudour E., 2003, Les terroirs viticoles, définitions, caractérisation, protection, Paris, Dunod.

\section{NOTES}

1. La moyenne du prix des terres en 2010 était dans le Lot de 3500 euros/ha alors que les domaines viticoles en état de production se négocient à plus de 50000 euros/ha, (avec les bâtiments et le matériel).

2. Il faut prendre conscience du hiatus qui existe entre l'aire AOC, plus de 20000 ha, et les 4000 et quelques qui sont plantées et déclarées chaque année en AOC. Ces 21708 ha ont été définis lors de la construction du VDQS en 1951 et confirmés par l'obtention de l'AOC en 1971, ils correspondent grosso modo à la superficie replantée après le phylloxéra, mais ne représentent plus ni le marché actuel, ni le potentiel en droits de plantation qui ne dépasse guère la superficie plantée actuelle. Plus de la moitié de la superficie AOC demeure aujourd'hui en bois et en garrigue, (E. Rouvellac, 2001).

\section{RÉSUMÉS}

Alliance parfois méconnue, la géographie et le droit possèdent une intersection facile à concevoir. Les territoires gérés par les sociétés font appel au droit et définissent des limites affirmant le passage d'un mode de gestion à un autre. Les appellations d'origine contrôlée en constituent un exemple de choix par les implications socio économiques que le droit peut avoir en structurant une appellation et un territoire viticole. Nous nous sommes intéressés au cas de l'aire $\mathrm{AOC}$ Cahors pour montrer la démarche à très grande échelle qui est nécessaire pour asseoir le droit sur des critères le moins contestables possible.

Sometimes underestimated alliance, the geography and the law possess an intersection easy to conceive. Territories managed by societies call to the law and define limits asserting the passage of a mode of management in an other one. Guaranteed vintage constitute an example of choice by the implications socioeconomic, which the law can have by structuring a guaranteed vintage and a winemaking territory. We were interested in the case of the area guaranteed vintage of 
Cahors to show in a very large-scale approach, which is necessary to base the law on the least questionable possible criteria.

\section{INDEX}

Keywords : legal geography, limits, guaranteed vintage, hierarchical organization, protected designation of origin Cahors

Mots-clés : géographie juridique, limites, appellations viticoles, hiérarchisation, AOC Cahors

\section{AUTEUR}

\section{ÉRIC ROUVELLAC}

Éric Rouvellac est maître de conférences HDR en géographie à l'université de Limoges, faculté des lettres et des sciences humaines au sein de l'UMR CNRS 6042 GEOLAB. Il travaille sur les questions de terroirs viticoles, leurs reconnaissances, leur cartographie, leur représentation et la manière dont les sociétés vitivinicole les utilisent.eric.rouvellac@unilim.fr 